\title{
A Value-Based Approach to Increase Breast Cancer Screening and Health-Directed Behaviors among American Indian Women
}

\author{
Marlene M. von Friederichs-Fitzwater • \\ Linda Navarro • Sandra L. Taylor
}

Received: 11 March 2010 /Accepted: 11 March 2010/Published online: 20 April 2010

(C) The Author(s) 2010. This article is published with open access at Springerlink.com

\begin{abstract}
American Indian/Alaska Native (AI/AN) women have the lowest cancer-screening rate of any ethnic or racial group; $\mathrm{AI} / \mathrm{AN}$ women in all regions are less likely than non-Hispanic white women to be diagnosed with localized breast cancer; and those $\mathrm{AI} / \mathrm{AN}$ women presenting with breast cancer have the lowest 5-year survival rate compared to other ethnic groups. This study found that cultural beliefs are more of a factor in mammography screening behavior than other barriers such as access; and that a more holistic educational intervention designed by $\mathrm{AI} / \mathrm{AN}$ women prompted individual intent and actions to seek mammograms among $\mathrm{AI} / \mathrm{AN}$ women $>40$ and to change unhealthy eating and sedentary lifestyles.
\end{abstract}

Keywords American Indian · Breast cancer

Mammography $\cdot$ Cultural barriers to screening

The American Indian and Alaska Native (AI/AN) population in California as of July, 2005 was 696,600, the highest

Presented at the 21st Annual Native Health Research Conference, Portland, OR, August 6, 2009

Supported by grant 12AB-3200 from the California Breast Cancer

Research Program

M. M. von Friederichs-Fitzwater $(\bowtie) \cdot$ S. L. Taylor

Davis School of Medicine, University of California,

UC Davis Cancer Center, 4501 X Street, Ste. 3016,

Sacramento, CA 95817, USA

e-mail: marlene.vonfriederichs-fitzwater@ucdmc.ucdavis.edu

L. Navarro

Turtle Health Foundation, Inc.,

Sacramento, CA, USA of any state. ${ }^{1}$ Since that population is distributed among more than 109 tribes, California's AI/AN population is well suited to the study of tribal differences and breast cancer risk reduction interventions. According to data included in the "Annual Report to the Nation on the Status of Cancer, 1973-1999," AI/ANs are not benefiting from the reduced cancer incidence rates documented for non-Hispanic whites (NHW) [1]. In particular, breast cancer is now the second leading cause of cancer-related deaths among AI/AN women [2], and mortality could be reduced by more than $30 \%$ if current recommendations for screening were followed by AI/AN women [2]. AI/ANs have the lowest cancer-screening rate of any ethnic or racial group, and those with breast cancer have the lowest 5-year survival rate [3-5]. In 2008, the Centers for Disease Control and Prevention reported that $\mathrm{AI} / \mathrm{AN}$ women in all regions of the country were less likely than NHW women to be diagnosed with localized disease [6]. In the 2001 California Health Interview Survey, researchers found that $10 \%$ of all eligible $\mathrm{AI} / \mathrm{AN}$ women in California have never had a mammogram [7]. They also found that only $54.6 \%$ of all AI/AN women in California aged 40 and older had received a mammogram in the past year, and $68.1 \%$ had received a mammogram in the past 2 years. Research has found that access to care, knowledge of the examination procedures (clinical breast examination and mammography), and cultural health beliefs were positively associated with breast cancer screening in multivariate analyses [2].

\footnotetext{
${ }^{1}$ US Census Bureau, July 1, 2005.
} 
Cultural beliefs/norms profoundly affect attitudes toward cancer, participation in screening, and compliance with treatment [8]. While little is known about cancer control strategies among $\mathrm{AI} / \mathrm{ANs}$, we do know that in some $\mathrm{AI} / \mathrm{AN}$ languages the literal translation for cancer is "the sore that never heals [9]," leading to the erroneous belief that cancer is by its nature incurable, and screening only reveals inevitable death. The stigma of cancer, especially among women, was a major obstruction to therapy in the early twentieth century among the broad American population and remains a factor in the $\mathrm{AI} / \mathrm{AN}$ communities today. Thus, conventional messages about cancer, screenings, and risk reduction may not receive wide attention or even acceptance.

$\mathrm{AI} / \mathrm{AN}$ health care beliefs and practices embody longstanding traditions and trust relationships that weave religion and medicine into one [10]. The Native American Cancer Research strongly encourages the inclusion of some form of spirituality within the cancer care of $\mathrm{AI} / \mathrm{ANs}$ and sees the value of including spiritual and cultural beliefs in any cancer control program [11]. The efficacy of a holistic perspective of health and health care integrated into a cultural approach to cancer prevention, control and care is the focus of this paper. The study examines changes in breast cancer knowledge and intended mammography screening attitudes among AI/AN women age 40 and older after exposure to a holistic educational/behavioral intervention integrating traditional values and beliefs with Western medicine practices.

\section{Methods}

The NCI-designated University of California, Davis, Cancer Center, an American Indian Advisory Council of 12 members from eight tribes and the Turtle Health Foundation, Inc., an $\mathrm{AI} / \mathrm{AN}$ nonprofit organization established to improve the health of all AI/ANs, partnered in 2004 to conduct focus groups/interviews with $\mathrm{AI} / \mathrm{AN}$ women to learn more about beliefs and knowledge of breast cancer, and to test a prototype of the resulting Mother's Wisdom Breast Health Program delivered via a multimedia, interactive DVD.

The results led to an 18-month pilot study with $161 \mathrm{AI} /$ AN women funded by the California Breast Cancer Research Program. Studies have focused on the first step to behavior change: changing knowledge levels, attitudes, and beliefs as well as intentions to change lifestyle (dietary changes and increased physical activity) and screening behaviors. We used a validated Knowledge, Attitudes, and Beliefs (KAB) multiple-choice survey in pre- and post-test design. The women completed the pre-test, watched, and discussed the Program DVD in Talking Circles, then took the post-test.
The DVD contains a menu of several short video segments on nutrition, exercise, breast structure and function, breast self-exams, mammography screening, breast cancer myths, and stories shared by AI/AN breast cancer survivors. "Rez Robics," featuring well-known AI/AN celebrities exercising to American Indian music and simple daily exercises that women could do at home or work was demonstrated by two members of the Council to encourage repeat usage of the DVD and retention of the content.

In the traditional AI/AN community, the medicine man or woman plays an important role in health care. For that reason, a medicine woman serves as co-chair of the American Indian Advisory Council, is a co-PI on the grants received, and co-authored this paper. She provides a welcome message on the Program DVD and assisted in presenting the Program to participants. Storytelling is also a unique and effective means of communicating important information in tribal communities and was used in the Program to convey information and experiences.

The use of Talking Circles is another strategy used that is drawn from $\mathrm{AI} / \mathrm{AN}$ heritage and is suited for disseminating health-related information. While Talking Circles can have an educational component, their real value is the caring and supportive environment that is created to openly discuss concerns and issues related to health and well-being [2].

We used the Steps-to-Behavior change (SBC) model because it identifies intermediate program effects and specifies indicators to measure the process of behavioral change as it unfolds [12]. The measurement of intermediate steps or sub-indicators provides opportunities for early assessment in time for corrective action in the intervention. We used indicators of individual behavior change through each step: knowledge, approval, intention, practice, and advocacy with the idea being that behavioral change is preceded by changes in attitudes, beliefs, and knowledge as well as by developing an intention to change behavior. The study focused on measuring changes in attitudes towards breast cancer, as well as awareness and knowledge, and intentions to change lifestyle and mammography behaviors. Because intention to change precedes behavioral change in the model, the focus of this study was on intentions before and after participating in the program.

The validated Knowledge, Attitudes, and Beliefs (KAB), multiple-choice instrument was used in pre- and posttesting. The instrument is adapted from the National Behavioral Risk Factor Surveillance System (USDHHS) [13] and modified versions of Behavioral Risk Factor Surveillance System questionnaires validated in $\mathrm{AI} / \mathrm{AN}$ populations and used on a Northern Plains reservation [14], as well as in urban AI/AN communities $[15,16]$. Besides items on knowledge and attitudes, other items inquire about demographics (age, marital status, children, education, income, and insurance type) and includes questions about 
the length of time since the woman's last clinical breast examination and last mammogram.

A third instrument focused on the technical aspects of the DVD (quality of audio, visuals and video; ease of navigation and use, and evaluation of content) included questions about intended lifestyle and screening behavioral changes and was administered following the DVD.

The research plan, survey, recruitment plan, and recruitment materials were approved by the University of California, Davis Institutional Review Board as well as by the American Indian Advisory Council and the Turtle Health Foundation, Inc.

Recruitment of the 161 AI women $\geq 40$ in our study was accomplished through (1) the efforts of the American Indian Advisory Council, (2) the California Rural Indian Health Board (CRIHB), (3) tribal health clinics, and other American Indian organizations. Additional activities were undertaken using booths, exhibits, and recruiting stations at tribal Pow Wows, at the National Congress of American Indians, tribal health gatherings, and at elder gatherings. AI women $\geq 40$ and older self-selected to participate and the PI followed up to determine eligibility, to consent participants, and to deliver the pre-intervention survey. A free copy of the DVD and an American Indian Ribbon of Life were given to all who participated.

\section{Results}

One hundred and sixty-one AI women participated from 18 tribes in California; 160 completed pre- and post-surveys and DVD evaluations; one completed only the DVD evaluation. About half were over age 50 and the remaining between 40 and 50. Seventy-five percent were married or previously married (separated, divorced, or widowed) and $81 \%$ had children. Ninety-three percent had graduated high school, but only $37.5 \%$ had a college degree. Eighty-seven percent had incomes less than $\$ 20,000$. Eighty-one percent had some form of medical insurance.

The primary research questions were as follows: 1) Did viewing the DVD change the knowledge, attitudes, and beliefs of AI women regarding breast health and breast cancer? 2) Did viewing the DVD change the intention of AI women to get a mammogram? 3) Was the DVD received favorably by AI women? 4) What demographic characteristics and beliefs distinguish women who had a recent mammogram and those who had not? 5) Did the participation in the Program change attitudes about lifestyle behaviors? 6) Did participation in the Program change intentions about having a mammogram; and actual mammography screening?

Seven questions on the pre- and post-survey assessed the participants' knowledge of breast cancer. We compared the percentage of correct answers before and after viewing the DVD with a paired-sample Wilcoxon test. In addition, we compared the percentage of women answering each of the questions correctly before and after viewing the DVD to determine if knowledge changes varied among questions. We also compared questions related to attitudes and beliefs before and after the DVD. McNemar's test was used for questions with a Yes/No answer, and a paired-sample Wilcoxon test was used for questions with scaled responses. A Bonferroni adjustment was used to maintain a 0.05 family-wise error rate.

Breast health knowledge increased significantly after viewing the DVD. Out of seven questions specifically related to breast health and breast cancer knowledge, the average percentage of correct responses significantly increased from 36 to $95 \%$ after viewing the DVD $(p<$ $0.0001)$. In addition, the overall percentage of correct answers increased significantly for every question (Table 1). Most notably, only $48 \%$ of the participants agreed that breast cancer detected early has a good chance of being cured before viewing the DVD, but all agreed with this assertion after viewing the DVD.

In addition to increasing knowledge, the DVD was effective in improving participant's understanding of the prognosis and treatment. The mean responses to the questions relating to beliefs about the prognosis and treatment significantly shifted to more favorable responses after the DVD for four of the five questions. Similarly, the percentage of women responding favorably to survey questions relating to the prognosis and treatment increased.

McNemar's test was used to determine if there was a significant change in the intention of women to get a mammogram after the DVD. To assess factors influencing change of intention to get a mammogram, we focused on women who responded that they did not intend to get a mammogram before viewing the DVD. We divided these women into two groups: 1) women who did not intend to get a mammogram within 1-2 years after viewing the DVD (No-No group) and 2) women who changed to intending to get a mammogram after viewing the DVD (No-Yes group). We used a $t$ test to test for differences in the mean change in percentage of correct answers to the seven knowledge questions between these two groups. The difference was calculated as the percentage of correct answers after viewing the DVD minus the percentage of correct answers before viewing the DVD. We evaluated differences in the mean change for each belief question between these groups with Wilcoxon tests. The change in beliefs was calculated as the response before viewing the DVD minus the response after viewing the DVD. A Bonferroni adjustment was used to maintain a 0.05 family-wise error rate.

Viewing the DVD significantly increased the proportion of women stating they intend to get a mammogram 
Table 1 Differences in responses to the Mother's Wisdom Breast Health Program Survey before and after viewing the Mother's Wisdom Breast Health DVD

\begin{tabular}{|c|c|c|c|}
\hline Survey item & $\begin{array}{l}\text { Before } \\
\text { DVD }\end{array}$ & After DVD & $p$ value (test statistic) \\
\hline \multicolumn{4}{|l|}{ Knowledge, \% correct } \\
\hline Women who have close relatives with breast cancer are more likely to get it & 21 & 90 & $p<0.0001\left(\chi^{2}=107, d f=1\right)^{\mathrm{a}}$ \\
\hline Bumping, bruising breasts leads to breast cancer & 19 & 92 & $p<0.0001\left(\chi^{2}=115, d f=1\right)^{\mathrm{a}}$ \\
\hline Touching, fondling, squeezing breasts leads to breast cancer & 58 & 95 & $p<0.0001\left(\chi^{2}=59, d f=1\right)^{\mathrm{a}}$ \\
\hline Women with large breasts are more likely to get breast cancer & 55 & 94 & $p<0.0001\left(\chi^{2}=62, d f=1\right)^{\mathrm{a}}$ \\
\hline Breast cancer found early has a good chance of being cured & 48 & 100 & $p<0.0001\left(\chi^{2}=84, d f=1\right)^{\mathrm{a}}$ \\
\hline Most breast lumps are breast cancer & 21 & 94 & $p<0.0001\left(\chi^{2}=100, d f=1\right)^{\mathrm{a}}$ \\
\hline Women 50 years or older are more likely to get breast cancer & 41 & 92 & $p<0.0001\left(\chi^{2}=82, d f=1\right)^{\mathrm{a}}$ \\
\hline \multicolumn{4}{|l|}{ Knowledge, mean (SE) } \\
\hline Obesity is a risk factor for breast cancer in post-menopausal women & $3.22(0.076)$ & $4.28(0.041)$ & $p<0.0001(W=4,450)^{\mathrm{b}}$ \\
\hline Age is the most significant risk factor for breast cancer in women & $2.37(0.073)$ & $4.37(0.041)$ & $p<0.0001(W=1,075)^{\mathrm{b}}$ \\
\hline \multicolumn{4}{|l|}{ Attitudes, $\%$ endorsed } \\
\hline Would get mammogram if health care provider recommended it & 94 & 94 & - \\
\hline Would be more likely to get mammogram if female technician did it & 80 & 87 & $p=0.002\left(\chi^{2}=10, d f=1\right)^{\mathrm{a}}$ \\
\hline Would get mammogram if had to travel more than 50 miles to get it & 52 & 60 & $p=0.01\left(\chi^{2}=6.26, d f=1\right)^{\mathrm{a}}$ \\
\hline $\begin{array}{l}\text { Would be more likely to come to women's cancer-screening clinic at an } \\
\text { Indian health clinic } \\
\text { Attitudes, mean (SE) }\end{array}$ & 70 & 78 & $p=0.0005\left(\chi^{2}=12.25, d f=1\right)^{\mathrm{a}}$ \\
\hline Thought about change of getting breast cancer in past month & $1.54(0.055)$ & $1.56(0.056)$ & $p=0.85(W=12,660)^{\mathrm{b}}$ \\
\hline Had thoughts about getting breast cancer affecting mood in past month & $1.06(0.019)$ & $1.06(0.019)$ & - \\
\hline $\begin{array}{l}\text { Had thoughts about getting breast cancer affecting performance of daily } \\
\text { activities }\end{array}$ & $1(0)$ & $1(0)$ & - \\
\hline \multicolumn{4}{|l|}{ Beliefs, mean (SE) } \\
\hline Breast cancer treatment is worse than the cancer itself & $2.52(0.088)$ & $1.77(0.056)$ & $p<0.0001(W=17,866)^{\mathrm{b}}$ \\
\hline If you have breast cancer, it is better not to know & $2.21(0.135)$ & $1.12(0.040)$ & $p<0.0001(W=16,757)^{\mathrm{b}}$ \\
\hline Breast cancer treatment many not be as bad if cancer is found early & $3.48(0.121)$ & $4.32(0.054)$ & $p<0.0001(W=9,376)^{\mathrm{b}}$ \\
\hline Compared with other health problems, breast cancer is not important & $1.06(0.019)$ & $1.06(0.019)$ & - \\
\hline If you get breast cancer, you will die from it. & $2.76(0.123)$ & $1.38(0.044)$ & $p<0.0001(W=19,009)^{\mathrm{b}}$ \\
\hline
\end{tabular}

${ }^{\mathrm{a}}$ McNemar's test

${ }^{\mathrm{b}}$ Paired sample Wilcoxon test

To maintain a family-wise error rate of rejection for $p<0.003$ based on Bonferroni adjustment for $n=17$ tests.

$(p<0.001)$ Among all participants, only $16(9 \%)$ stated that they intended to get a mammogram in 1 to 2 years prior to viewing the DVD. After viewing, this number increased to 119 (74\%). About 20\% (33) of the women did not intend to get a mammogram prior to viewing the DVD and did not change their intention after viewing it.

Women who changed from not intending to get a mammogram to intending to get one after the DVD had much higher education levels and greater income than those who did not change intention. Time since last mammogram differed markedly between those who changed intention and those who did not. The majority who changed had a mammogram within the last 2 years $(69.6 \%)$ while most women $(75.7 \%)$ who did not change intention had not had one in the last 2 years. Finally, a large majority (146) responded on the DVD evaluation that they would be likely to get a mammogram whereas somewhat fewer (119) responded on the breast health survey that they intended to get a mammogram years after viewing the DVD.

Changes in knowledge and some beliefs distinguished between women that changed their intention to get a mammogram. The change in percentage of correct answers before and after viewing the DVD differed significantly between the two groups $(p=0.01)$ suggesting that with increased knowledge, women are more likely to change their intention to get a mammogram. Two beliefs differed significantly between women that did not change intention and those that did. First, women who changed had significantly greater movement toward agreeing that treatment may not be as bad if the cancer is found early. 
Secondly and somewhat counter-intuitively, women who changed showed less movement toward disagreeing with the question "If you get breast cancer, you die from it."

We evaluated differences in knowledge, attitudes, and beliefs before viewing the DVD between those who had had a recent mammogram and those who had not. Agerelated risk was the only knowledge question for which correct responses differed between these two groups. Significantly, more of the women who had a recent mammogram regarded age to be a significant risk factor. Women who did not have a recent mammogram were more likely to prefer to have a mammogram administered by a female technician and more likely to prefer to have screening available at an Indian health clinic than women who had a recent mammogram. This finding may suggest that women who have had a recent mammogram believe it is important to be screened regardless of the clinical environment. Unexpectedly, significantly more women who had a recent mammogram did not believe that treatment would not be as bad if breast cancer was detected early. Consistent with this view, women who had a recent mammogram disagreed more strongly with the statement that death was inevitable given a breast cancer diagnosis.

About 1 year after completion of the Program, 158 women responded to a follow-up telephone survey asking if they had received a mammogram. We used McNemar's test to evaluate if women who stated that they intended to get a mammogram subsequently had a mammogram and found a significant association between stating an intention to get a mammogram and actually getting one $(p<0.001)$. Of 118 women stating that they intended to get a mammogram, $95 \%$ obtained a mammogram. Interestingly, of those who did not intend to get a mammogram, subsequently $80 \%$ did obtain one.

We evaluated if having health insurance influenced whether a women had a mammogram in addition to her stated intention and used a logistic regression to model whether a woman had a mammogram as a function of her intention and whether she had insurance. Having insurance did not significantly influence the decision.

A separate survey was also administered to assess opinions about the DVD immediately after viewing and after completing the post-intervention questionnaire. One woman completed the DVD evaluation, but did not complete the pre- and post-intervention questionnaire, so these results have 161 responses. The major findings are as follows:

- The DVD received very favorable ratings overall. For all questions, a large portion of the women rated the material as very good/excellent. The program was strongly regarded as being culturally appropriate and appealing with 143 of the 161 respondents strongly agreeing and 18 agreeing. A large majority (143) strongly agreed that the DVD content would help them in making healthier diet and exercise choices.

- All women agreed that they would be likely to get a mammogram after viewing the DVD and most (146) strongly agreed.

- A large majority (149) responded that they would share the DVD and other information with others. The fact that women responded favorably to the DVD and reported that they would share it with others, suggests that the DVD could facilitate transitioning women from knowledge to approval, the second step to behavioral change.

Our study also demonstrated the value of a more holistic approach to breast health, including the importance of healthy eating, reducing obesity, and increasing physical activity. These segments were very well received as reported in the DVD evaluation questionnaire. A large majority, $89 \%$, (143) strongly agreed that the content would help them make healthier diet and exercise choices and $11 \%$ (18) agreed it would help them.

We also learned that traditionality issues (social, cultural) influenced cancer-screening behavior more than other factors and were similar across tribes. For example:

- $97 \%$ reported that tribal community influenced health decisions

- $91 \%$ reported discomfort with raising the topic of cancer within the family or tribal community

- $\quad 94.5 \%$ agreed that awareness and knowledge levels were significant predictors of cancer-screening behaviors

- $98 \%$ reported that health-related information from tribal leaders and elders was credible and honored

\section{Discussion}

Mammography is the primary method used for breast cancer screening. However, despite multiple educational campaigns, adherence to recommended screening practices is still below acceptable levels [17]. Among AI/AN women, screening rates are lower than other racial/ethnic groups. Our 18-month study proved our hypotheses that AI/AN women would be receptive to a holistic educational intervention that is culturally sensitive, interactive, and visual and it would increase their intention to have mammography screening as well as motivate them to adopt healthier lifestyles. Thus, using the SBC model demonstrated that participation in our Mother's Wisdom Breast Health Program led to changes in attitudes, beliefs, and knowledge and prompted individual intentions to seek mammograms and to make lifestyle changes. There was a 
significant increase in completing mammography screening following participation.

The three-generation extended family is common to many tribes [18]. This common family structure and lengthy formative discussions on the role of women, particularly mothers, in health care decision-making in AI/ AN communities played a part in the naming the Program Mother's Wisdom Breast Health Program. Many tribes are matrilineal, children belong to the mother's clan, and children are highly valued in these families. Women are respected, influential and the "keepers of wisdom" for families, according to the women who guided this project and participants will share the Program with other female family and community members.

This study succeeded in recruiting more participants than planned, included women from a variety of tribes, and provided new insight on strategies for developing breast cancer risk reduction interventions. One limitation is that we only measured intentions to change lifestyle and screening behaviors and self-reports on completion of mammography screening, not actual numbers of mammograms. Another possible limitation was in including women who had a mammogram within the past year, but we wanted to learn whether they understood the need for annual mammography, and the results indicate that most women did not understand the need for regular screening.

\section{Conclusion}

This study demonstrated the importance of not only incorporating cultural beliefs and values in increasing mammography screening rates as well as the need to use a more holistic approach to engage $\mathrm{AI} / \mathrm{AN}$ women to encourage them to frequently refer back to the intervention for message retention. A 3-year study has been proposed to measure actual lifestyle and clinical screening behavior changes among $\mathrm{AI} / \mathrm{AN}$ women $\geq 40$ and older and we expect to be able to demonstrate that participation in the Mother's Wisdom Breast Health Program results in sustained changes in lifestyle and breast cancer-screening behaviors.

Open Access This article is distributed under the terms of the Creative Commons Attribution Noncommercial License which permits any noncommercial use, distribution, and reproduction in any medium, provided the original author(s) and source are credited.

\section{References}

1. Edwards BK, Holly HL, DiFies LAG et al (2002) Annual report to the nation on the status of cancer, 1973-1999: featuring implications of age and aging on the US cancer burden. Cancer 94:2766-2792

2. Risendal B, Roe D, DeZapien J, Papenfuss M, Giuliano A (1999) Influence or health care, cost, and culture on breast cancer screening: issues facing urban American Indian women. Prev Med 29(6):501-509

3. Paskett ED, Tatum C, Rushing J et al (2004) Racial differences in knowledge, attitudes, and cancer screening practices among a triracial rural population. Cancer 101:2650-2658

4. Breast Care in American Indian Women (2000) Breast cancer in New Mexico: a handbook for health care providers. University of New Mexico, 14-15

5. Department of Health and Human Services (1992) PHS, NIH, NCXI. Report of the special action committee, 1992: Program initiatives related to minorities; the underserved and persons aged 65 and over. Washington, DC: government printing offices. Appendices A, B, C

6. Centers for Disease Control and Prevention, Division of Media Relations (2008) Report shows high regional lung and colorectal rates in American Indians and Alaska Natives in the United States, 1999-2004

7. Seals BF, Burhansstipanov L, Satter DE, Chia J, Gatchell M (2006) California American Indian and Alaska Natives tribal groups' care access and utilization of care: policy implications. J Cancer Educ S221(1):S15-S21

8. Liora N (1999) Cultural views of cancer around the world. Cancer Nurs 22(1):39-45

9. Native Americans and Cancer. Retrieved from http://natamcancer. org/page12.html on September 4, 2008

10. Cesario SK (2001) Care of the Native American women: strategies for practice, education and research. JOGNN 30 (1):13-19

11. Burhansstipanov L. Retrieved from http://litsite.uaa.alaska.edu/ healing/burhan.html on April 6, 2008

12. von Friederichs-Fitzwater MM (2006) Addressing cultural and tribal issues in breast cancer. J Cancer Educ Supplement, 21(2)

13. Department of Health and Human Services (USDHHS) (1999) Behavioral Risk Factor Surveillance System Summary. Retrieved from http://www.cdc.gov/brfss/ti-docs.htm

14. Welty TK, Zephier N, Schweigman K, Blake B, Leonardson G (1993) Cancer risk factors in three Sioux tribes. Use of the Indianspecific health risk appraisal for data collection and analysis. Alaska Med 35(4):265-272

15. Burhansstipanov L, Dignan MB, Wound DB, Tenney M, Vigil G (2000) Native American recruitment into breast cancer screening: the NAWWA project. J Cancer Educ 15(1):28-32

16. Wampler NS, Ryschon R, Manson SM, Buchwald D (2006) Knowledge, attitudes and beliefs regarding breast cancer among American Indian women from the Northern Plains. J Appl Gerontology 25(1):445-595

17. Saywell RM, Champion VL, Skinner CS, Menon U, Daggy J (2004) A cost-effectiveness comparison of three tailored interventions to increase mammography screening. J Women's Health 13(8):909-918

18. Seideman R, Jacobson S, Primeaux M, Burns P, Weatherby F (1996) Assessing American Indian families. MCN Am J Matern Child Nurs 21:274-279 